\title{
The effect of time-dependent macromolecular crowding on the kinetics of protein aggregation: a simple model for the onset of age-related neurodegenerative disease
}

\section{Allen P. Minton*}

Section on Physical Biochemistry, Laboratory of Biochemistry and Genetics, National Institute of Diabetes and Digestive and Kidney Diseases, National Institutes of Health, US Department of Health and Human Services, Bethesda, MD, USA

\section{Edited by:}

Francesco Piazza, University of

Orléans, France

Reviewed by:

Alexander Raymond Small,

California State Polytechnic

University, Pomona, USA

Davide Marenduzzo, University of

Edinburgh, UK

*Correspondence:

Allen P. Minton, National Institutes of Health, Building 8, Room 226,

Bethesda, MD 20892-0830, USA

e-mail:minton@helix.nih.gov
A linear increase in the concentration of "inert" macromolecules with time is incorporated into simple excluded volume models for protein condensation or fibrillation. Such models predict a long latent period during which no significant amount of protein aggregates, followed by a steep increase in the total amount of aggregate. The elapsed time at which these models predict half-conversion of model protein to aggregate varies by less than a factor of two when the intrinsic rate constant for condensation or fibril growth of the protein is varied over many orders of magnitude. It is suggested that this concept can explain why the symptoms of neurodegenerative diseases associated with the aggregation of very different proteins and peptides appear at approximately the same advanced age in humans.

Keywords: excluded volume, protein condensation, protein fibrillation, Alzheimer's Dementia, Parkinsonism

\section{INTRODUCTION}

Several neurodegenerative diseases, most prominent among them Alzheimer's Dementia and Parkinsonism, are extremely rare in individuals under the age of about sixty, but the incidence rises sharply above that age, reaching $10-25 \%$ at the age of eighty $[1,2]$. The root cause of age-related incidence has been the subject of much conjecture (Google "aging and neurodegeneration") but no solid consensus has been formed. The purpose of this communication is to suggest a common mechanism underlying the age-related onset of a variety of neurodegenerative diseases based upon the effect of excluded volume on the kinetics of protein aggregation.

The model to be developed here is based upon the notion that most if not all proteins are subject to a gradual process of degradation leading to a loss of solubility and consequent biological function. Under laboratory conditions thought to reflect physiological conditions, degradation may be imperceptibly slow on the time scale of an ordinary experiment. However, it is a common experience that proteins in purified solutions left at room temperature for extended periods will begin to precipitate, and that addition of small amounts of destabilizing agents can significantly accelerate the loss of function or formation of various types of aggregates.

It was shown long ago that the addition of a moderately high concentration of functionally unrelated polymers or proteins can significantly reduce the solubility of a variety of trace proteins [3-5], and quantitative models based upon the concept of excluded volume were shown to account semiquantitatively for the observed dependence of solubility on the concentration of added polymer or protein were introduced $[3,4,6,7]$.
More recently, studies carried out in several laboratories [812] revealed that the addition of significant concentrations of functionally unrelated polymers or proteins can greatly accelerate the formation of fibrillar protein aggregates. A simple model based upon excluded volume theory was shown to quantitatively account for the dependence of the kinetics of amyloid fiber formation upon the concentration of added polymer [10].

At the same time, gerontological studies have revealed a gradual reduction in the water content of aging tissue, including brain tissue [13-16], which is equivalent to an increase in the total macromolecular concentration in the aqueous compartments of these tissues.

In the following section we review simple models for the effect of excluded volume ("crowding") upon the kinetics of solubility loss and fiber formation. Next we introduce a timedependent increase in the concentration of an additional inert volume-excluding substance (termed "crowder"), and show that this leads to a highly cooperative appearance of aggregate. The models developed here provide a possible explanation of why aggregates of different proteins with widely disparate intrinsic stabilities may accumulate on a comparable time scale in aging individuals.

\section{MODELS}

The models to be summarized below are based upon highly simplified hard particle representations of molecular size and shape, which have been found useful for semi-quantitative estimation of the effect of crowding upon the free energies, equilibrium, and rate-constants of reactions in crowded solutions [17-19]. A complementary description of the crowding effect termed "depletion attraction" has been presented [20] but so far has not been 
utilized to quantify the effect of crowding on chemical equilibria and kinetics.

\section{CONDENSATION}

The reaction to be modeled is $P$ (solution) $\rightleftarrows P$ (aggregate). $P$ in solution is assumed to be monomeric, and the aggregate is assumed to be thermodynamically equivalent to a condensed phase. The thermodynamic solubility constant is given by

$$
K_{s}^{0}=\frac{1}{a_{s}}=\frac{1}{\gamma_{s} c_{s}}
$$

where $a_{s}, \gamma_{s}$, and $c_{s}$ respectively denote the thermodynamic activity, activity coefficient, and concentration of soluble protein in equilibrium with the condensed phase. We then define the effective solubility constant [21]

$$
K_{s} \equiv \frac{1}{c_{s}}=K_{s}^{0} \gamma_{s}
$$

For transition-state rate limited (i.e., slow) association reactions, crowding affects primarily the association rate constant [21], so we may write

$$
\ln \left(k_{a} / k_{a}^{0}\right) \cong \ln \left(K_{s} / K_{s}^{0}\right)=\ln \gamma_{s}
$$

where $k_{a}^{0}$ denotes the value of the association rate constant in dilute (ideal) solution. We represent the soluble protein as an equivalent hard sphere with radius $r_{s}$ and the background environment as a fluid containing a volume fraction $\phi$ of uniform hard spheres ("crowders") with radius $r_{c}$. Scaled particle theory [22] then yields the approximate expression

$$
\ln \gamma_{s}=\ln (1-\phi)+A_{1} z+A_{2} z^{2}+A_{3} z^{3}
$$

where

$$
\begin{aligned}
A_{1} & =R^{3}+3 R^{2}+3 R \\
A_{2} & =3 R^{3}+4.5 R^{2} \\
A_{3} & =3 R^{3} \\
z & =\frac{\phi}{1-\phi}
\end{aligned}
$$

and $R \equiv r_{s} / r_{c}$.

We assume that the condensed phase (aggregate) is essentially insoluble, and that the rate of dissolution may be neglected. The kinetics of aggregation are then described by the following firstorder rate equation:

$$
\frac{d f_{s}}{d t}=-k_{a} f_{s}
$$

where $f_{s}$ denotes the mass fraction of soluble protein, and the fraction of aggregated protein is just $1-f_{s}$.

\section{FIBRILLATION}

The association reaction to be modeled is the addition of monomer to the end(s) of a growing fibrillar aggregate: $P_{1}+$ $P_{n} \rightleftarrows P_{n+1}$. The thermodynamic equilibrium constant is given by

$$
K_{n}^{0}=\frac{a_{n+1}}{a_{1} a_{n}}=\frac{\gamma_{n+1} c_{n+1}}{\gamma_{1} c_{1} \gamma_{n} c_{n}}
$$

and the effective equilibrium defined in terms of concentrations is then given by

$$
K_{n} \equiv \frac{c_{n+1}}{c_{1} c_{n}}=K_{n}^{0} \frac{\gamma_{1} \gamma_{n}}{\gamma_{n+1}}
$$

As before, the monomer is modeled as an equivalent hard sphere of radius $r_{1}$, and its activity coefficient calculated according to Equations (4-6), with $R=R_{1} \equiv r_{1} / r_{c}$. The oligomeric fibrils $P_{n}$ and $P_{n+1}$ are modeled as hard spherocylinders (cylinders capped with hemispheres on each end) with radius $r_{s c}$ and the ratios of cylindrical length to diameter $L_{n}$ and $L_{n+1}$ respectively. The activity coefficient of an isolated spherocylinder in a fluid containing volume fraction $\phi$ of uniform hard spheres of radius $r_{c}$ is estimated using Equations $(4,6)$, with

$$
\begin{aligned}
& A_{1}=R^{3}+3 R^{2}+3 R+1.5 L\left(R^{2}+2 R+1\right) \\
& A_{2}=3 R^{3}+4.5 R^{2}+4.5 L\left(R^{2}+R\right) \\
& A_{3}=3 R^{3}+4.5 L R^{2}
\end{aligned}
$$

where $R=R_{s c} \equiv r_{s c} / r_{c}[23]$.

As in the case of condensation, it is assumed that crowding affects primarily the association rate constant, so that

$$
\ln \left(k_{a} / k_{a}^{0}\right) \cong \ln \left(K_{n} / K_{n}^{0}\right)=\ln \gamma_{1}+\ln \gamma_{n}-\ln \gamma_{n+1}
$$

We may write

$$
\ln \gamma_{n+1}=\ln \gamma_{n}+\frac{d \ln \gamma}{d n}=\ln \gamma_{n}+\frac{d \ln \gamma}{d L} \frac{d L}{d n}
$$

It follows from equations $[10 \mathrm{a}-\mathrm{c}]$ that

$$
\begin{aligned}
\frac{d \ln \gamma_{s c}}{d L}= & 1.5\left(R_{s c}^{2}+2 R_{s c}+1\right) z \\
& +4.5\left(R_{s c}^{2}+R_{s c}\right) z^{2}+4.5 R_{s c}^{2} z^{3}
\end{aligned}
$$

Assuming conservation of volume upon association, we obtain

$$
\frac{d L}{d n}=\frac{2}{3}\left(\frac{r_{1}}{r_{s c}}\right)^{3}
$$

Inspection of Equations (12-14) reveals that $\ln \gamma_{n+1}-\ln \gamma_{n}$, equal to the excess free energy of adding a monomer to an oligomer, is independent of the size of the oligomer. This result agrees with our intuition, since fiber growth only occurs at the ends of the oligomer. Then Equation (11) may be rewritten as

$$
\ln \left(k_{a} / k_{a}^{0}\right)=\ln \gamma_{1}-\frac{d \ln \gamma_{s c}}{d L} \frac{d L}{d n}
$$


where $\ln \gamma_{1}$ is calculated using Equations (4-6) with $R=R_{1}$, and $d \ln \gamma_{s c} / d L$ and $d L / d n$ are calculated using Equations $(13,14)$ respectively.

As in the case of the condensation model, we shall assume that the linear fibrils are essentially insoluble, so that the dissociation rate may be neglected relative to the association rate. Then the rate of addition of monomer to oligomer will be given by the first order rate expression

$$
\frac{d f_{1}}{d t}=-k_{a} f_{1}
$$

where $f_{1}$ denotes the mass fraction of monomer, and the mass fraction of fibril is just $1-f_{1}$.

\section{TIME-DEPENDENCE OF CROWDING}

The concentration of soluble protein in various regions of human brain has been reported to increase roughly linearly with age [13], in some regions doubling over a lifespan of 80 years. We therefore model the total volume fraction of "crowder" as a linear function of time:

$$
\phi=\phi_{0}+\beta t
$$

Parameswaran et al. [16] measured the fraction of volume excluded to albumin in rat mesenteric tissue as a function of the age of the rat, and reported that this fraction increased from 20 to $40 \%$ over the lifetime of the rat (650 days). We shall use the results cited above as a qualitative basis for positing a possible linear increase in volume fraction of crowder from 0.2 to 0.4 over the 80 year lifespan of a human, i.e., $\phi_{0}=0.2$ and $\beta=0.0025 \mathrm{yr}^{-1}$. Simulations were carried out varying each of these parameters about the values cited.

Calculation of the mass fraction of aggregated protein according to the solubility model was performed by numerical integration of Equation (7) with a time-dependent value of $k_{a}$ calculated according to Equations $(3-5,17)$, using the differential equation solver ODE15s in MATLAB v. 2013b (Mathworks, Natick, $\mathrm{MA})$. The corresponding calculation carried out according to the fibrillation model was performed by numerical integration of Equation (16) with a time-dependent value of $k_{a}$ calculated according to Equations $(13,14,15)$.

\section{RESULTS AND DISCUSSION}

The dependence of the mass fraction of polymer on elapsed time, calculated using the condensation and fibrillation models with various parameter sets shown in the figure caption, are plotted in Figures 1A,B. The dashed reference curve in panel A represents the classical decaying exponential solution to the first-order rate Equation (7) with $k_{a}$ equal to a constant value of 0.1 . Results obtained from both models are qualitatively similar. Each curve representing the calculated dependence of $f_{p}$ on elapsed time may be characterized by two parameters: $t_{50}$, the time at which half of the protein is aggregated, and a kinetic cooperativity parameter, $\alpha$, a measure of the steepness of the curve at $t_{50}$, defined according to Hall and Mnton [24]

$$
\alpha=\frac{\left(d \ln f_{p} / d \ln t\right)_{t=t_{50}}}{\ln 2}
$$

The value of $\alpha$ so defined is identically equal to 1 for the time dependence of $f_{p}$ obtained from solution of Equation (7) with a constant (time-independent) value of $k_{a}$ (for example, the reference curve plotted in Figure 1A), and increases with the steepness of the dependence of $f_{p}$ on $t$. General properties of the model may be easily deduced upon inspection of correlations between the various input parameters and the output parameters $t_{50}$ and $\alpha$. It follows from Equations $(16,18)$ that

$$
\frac{\alpha}{t_{50}}=\frac{k_{a, 50}}{\ln 2}
$$

where $k_{a, 50}$ is the value of $k_{a}$ at $t=t_{50}$.

In Figure 2A the dependence of $f_{p}$ on elapsed time is plotted for three simulations carried out with the condensation model with the parameters indicated in the Figure caption. These parameters were selected to provide significantly different rates of increase of $k_{a}$ with time, as shown in Figure 2B, but similar values

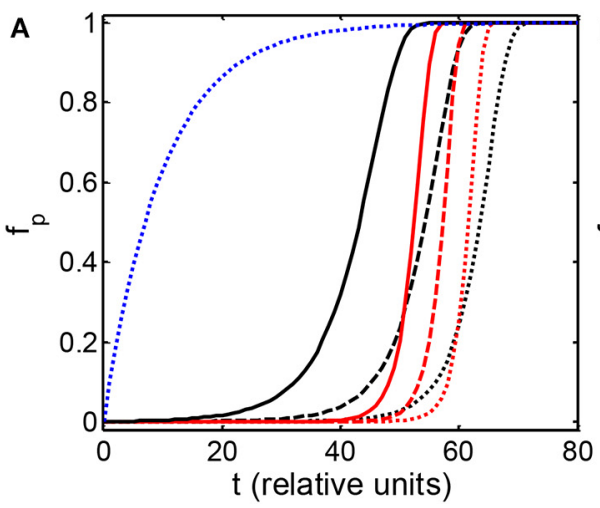

FIGURE 1 | Dependence of the mass fraction of polymer upon elapsed time, calculated according to the condensation model (A) and the fibrillation model (B) as described in the text. Parameters associated with

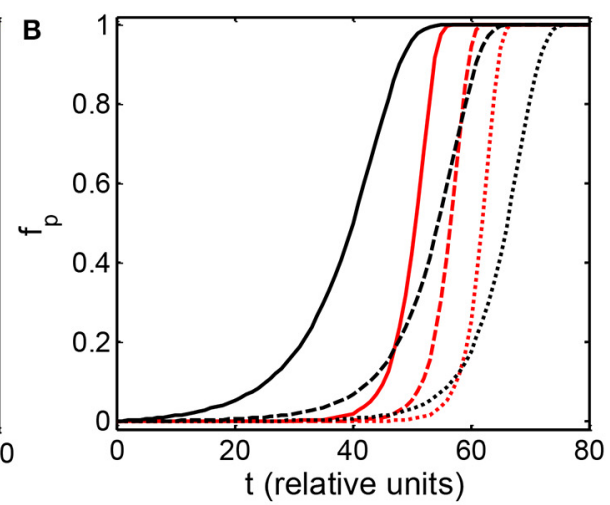

each simulation are presented in Table 1. The blue dotted curve in (A) is the solution to the first order rate Equation (7) with a constant value of log $k_{a}=-1$. 
of $t_{50}$. The results of comparable simulations carried out using the fibrillation model are qualitatively similar and are therefore not shown. It is evident that the delay time preceding the onset of the appearance of significant quantitities of polymer, and the rapidity of significant accumulation of polymer, as reflected in the value

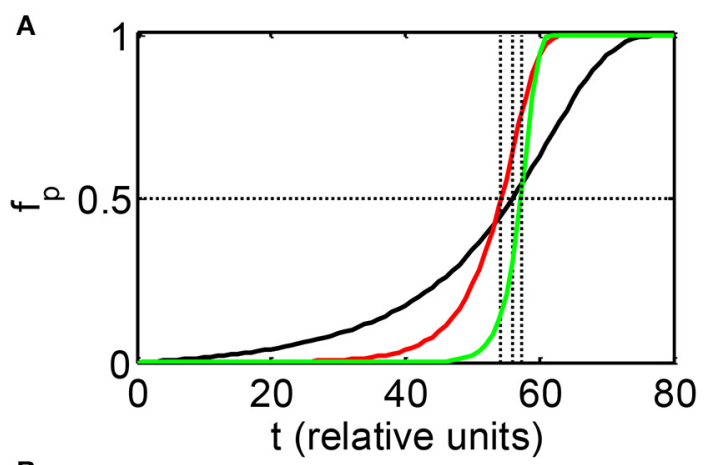

B

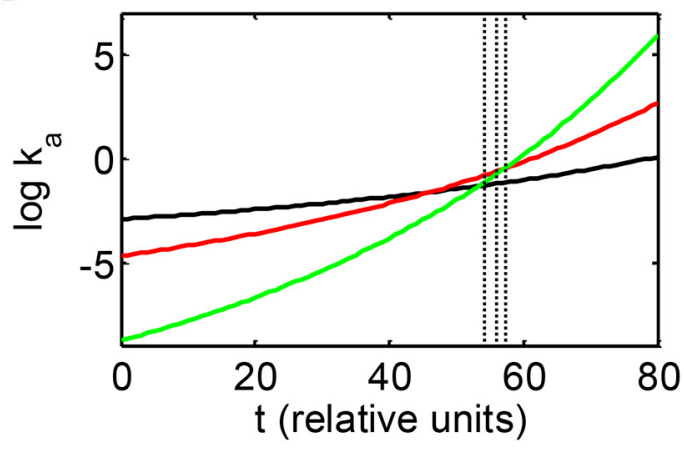

FIGURE 2 | Dependence of the mass fraction of polymer $(A)$ and the logarithm of the association rate constant (B), calculated according to the condensation model as described in the text. Parameters associated with each simulation are presented in Table 2. Vertical dotted lines indicate the values of $t_{50}$ associated with each simulation. of $\alpha$, are both strongly correlated with the rate of increase of $k_{a}$, and thus the rate of increase of volume occupancy by crowder [cf. Equations (3-5)]. It should also be noted that simulations yielding a relatively abrupt onset of the appearance of polymer (i.e., a high value of $\alpha$ ), with very similar values of $t_{50}$ (e.g., curves b and $\mathrm{c}$ of Figure 2A) may be obtained for values of $k_{a}^{0}$ differing by several orders of magnitude.

We therefore conclude that, independent of the particular model or details of the simulation, the apparently sudden accumulation of significant amounts of aggregate following a long delay is a consequence of a rate constant for polymer growth that is initially undetectably small, but increases at least exponentially with time. These conditions are met in a wide variety of simulations reflecting broad variation in initial rates of polymer growth, polymer geometry and size, and postulated temporal gradients of volume occupancy by an inert crowder.

The model for fibrillation presented here does not take into account the potential fragmentation of growing fibrils [25]. The rate of fragmentation would be expected to increase with the average size of growing fibrils and hence an increase in the number of growth sites with time, leading to a potentially cooperative accumulation of fibrillated protein with time [26]. An increasingly crowding-accelerated rate of addition of monomer to an increasing number of growth sites would enhance the

Table 2 | Input and output parameters characterizing each simulation, calculated using the condensation model, plotted in Figures 2A,B.

\begin{tabular}{lccccc}
\hline Color & \multicolumn{2}{c}{ Input parameters } & & \multicolumn{2}{c}{ Output parameters } \\
\cline { 2 - 3 } \cline { 5 - 6 } & $\boldsymbol{R}$ & $\log k_{\boldsymbol{a}}^{\mathbf{0}}$ & & $\boldsymbol{t}_{\mathbf{5 0}}$ & $\boldsymbol{\alpha}$ \\
\hline Black & 1 & -4 & & 56.0 & 4.9 \\
Red & 1.5 & -7 & & 54.3 & 12.4 \\
Green & 2 & -13 & & 57.3 & 29.0 \\
\hline
\end{tabular}

Table 1 | Input and output parameters characterizing each simulation plotted in Figures 1A,B.

\begin{tabular}{|c|c|c|c|c|c|c|c|c|}
\hline \multirow[t]{2}{*}{ Panel:model } & \multirow[t]{2}{*}{ Color } & \multirow[t]{2}{*}{ Line type } & \multicolumn{3}{|c|}{ Input parameters } & \multirow[t]{2}{*}{$\log k_{a}^{0}$} & \multicolumn{2}{|c|}{ Output parameters } \\
\hline & & & $\boldsymbol{R}$ & $R_{1}$ & $R_{s c}$ & & $t_{50}$ & $\alpha$ \\
\hline \multirow[t]{6}{*}{ A: condensation } & Black & Solid & 1.5 & - & - & -6 & 43.2 & 8.1 \\
\hline & & Dash & & & & -7 & 54.3 & 12.4 \\
\hline & & Dot & & & & -8 & 63.6 & 17.2 \\
\hline & Red & Solid & 2.0 & - & - & -11 & 47.3 & 20.0 \\
\hline & & Dash & & & & -12 & 52.5 & 24.4 \\
\hline & & Dot & & & & -13 & 57.3 & 29.0 \\
\hline \multirow[t]{6}{*}{ B: fibrillation } & Black & Solid & - & 1.5 & 3 & -5 & 40.0 & 5.6 \\
\hline & & Dash & & & & -6 & 54.5 & 9.8 \\
\hline & & Dot & & & & -7 & 66.2 & 14.4 \\
\hline & Red & Solid & - & 2 & 4 & -10 & 50.7 & 18.6 \\
\hline & & Dash & & & & -11 & 56.6 & 23.2 \\
\hline & & Dot & & & & -12 & 62.0 & 28.0 \\
\hline
\end{tabular}


cooperative appearance of fibril exhibited by the model presented here, thus strengthening the qualitative conclusions derived from this model.

While the models proposed above are highly speculative, we believe that they may have physiological relevance for the following reasons. The appearance of neurodegenerative diseases is relatively abrupt and occurs at an advanced age, except in the case of unstable mutant proteins. There have been many hypotheses proposed relating the aggregation of a protein or peptide associated with a particular disease to the ensuing neuronal damage and death $[27,28]$, including loss of essential native protein function and toxicity of oligomers or large aggregates. The models proposed here suggest that if neurons are particularly susceptible to water loss and the concomitant increase in intracellular crowding with aging, then many neuronal proteins, independent of their intrinsic stability, would at some point abruptly appear to lose solubility as their respective rates of condensation or fiber formation begin to rapidly accelerate. Which protein first becomes substantially insoluble, and thus identified as the cause of a particular infirmity, depends upon the time-dependent macromolecular composition of a particular cell or tissue type, and is therefore subject to variation between individuals.

\section{ACKNOWLEDGMENTS}

The author thanks Drs. Peter McPhie and Travis Hoppe (NIH) for helpful comments on an early draft of this report. This work was supported by the Intramural Research Program of the National Institute of Diabetes and Digestive and Kidney Diseases

\section{REFERENCES}

1. Hebert LE, Scherr PA, Beckett LA, Albert MS, Pilgrim DM, Chown MJ, et al. Age-specific incidence of Alzheimer's Disease in a community population. J Am Med Assoc. (1995) 273:1354-9. doi: 10.1001/jama.1995.035204 10048025

2. Gurland BJ, Wilder DE, Lantigua R, Stern Y, Chen J, Killeffer EH, et al. Rates of dementia in three ethnoracial groups. Int J Geriatric Psychiatry (1999) 14:481-93.

3. Laurent TC. The interaction between polysaccharides and other macromolecules VI. Further studies on the solubility of proteins in dextran solutions. Acta Chem Scand. (1963a) 17:2664-8. doi: 10.3891/acta.chem.scand.17-2664

4. Laurent TC. The interaction between polysaccharides and other macromolecules. The solubility of proteins in the presence of dextran. Biochem J. (1963b) 89:253-7.

5. Atha DH, Ingham KC. Mechanism of precipitation of proteins by polyethylene glycols. Analysis in terms of excluded volume. J Biol Chem. (1981) 256:12108-117.

6. Laurent TC, Ogston AG. The interaction between polysaccharides and other macromolecules. 4 . The osmotic pressure of mixtures of serum albumin and hyaluronic acid. Biochem J. (1963) 89:249-53.

7. Ogston AG. On interaction of solute molecules with porous networks. J Phys Chem. (1970) 74:668-9. doi: 10.1021/j100698a032

8. Wilf J, Gladner JA, Minton AP. Acceleration of fibrin gel formation by unrelated proteins. Thromb. Res. (1985) 37:681-8. doi: 10.1016/00493848(85)90197-5

9. Drenckhahn D, Pollard TD. Elongation of actin filaments is a diffusion-limited reaction at the barbed end and is accelerated by inert macromolecules. J Biol Chem. (1986) 261:12754-8.

10. Hatters DM, Minton AP, Howlett GJ. Macromolecular crowding accelerates amyloid formation by human apolipoprotein C-II. J Biol Chem. (2002) 277:7824-30. doi: 10.1074/jbc.M110429200

11. Shtilerman MD, Ding TT, Lansbury PT, Jr. Molecular crowding accelerates fibrillization of alpha-synuclein: could an increase in the cytoplasmic protein concentration induce Parkinson's disease? Biochemistry (2002) 41:3855-60. doi: 10.1021/bi0120906

12. Uversky VN, Cooper EM, Bower KS, Li J, Fink AL. Accelerated alphasynuclein fibrillation in crowded milieu. FEBS Lett. (2002) 515:99-103. doi: 10.1016/S0014-5793(02)02446-8

13. Naber D, Korte U, Krack K. Content of water-soluble and total proteins in the aging human brain. Exp Geront. (1979) 14:59-63. doi: 10.1016/05315565(79)90010-X

14. Nagy I, Nagy K, Nagy V, Kalmar A, Nagy E. Alterations in total content and solubility characteristics of proteins in rat brain and liver during aging and centrophenoxine treatment. Exp Geront. (1981) 16:229-40. doi: 10.1016/05315565(81)90018-8

15. Nagy IZ, Nagy K, Lustyik G. Protein and water contents of aging brain. Exp Brain Res. Suppl. (1982) 5:118-22. doi: 10.1007/978-3-642-68507-1_15

16. Parameswaran S, Barber BJ, Babbitt RA, Dutta S. Age-related changes in albumin-excluded volume fraction. Microvasc Res. (1995) 50:373-80. doi: 10.1006/mvre.1995.1065

17. Hall D, Minton AP. Macromolecular crowding: qualitative and semiquantitative successes, quantitative challenges. Biochim Biophys Acta (2003) 1649:127-39. doi: 10.1016/S1570-9639(03)00167-5

18. Minton AP. The effective hard particle model provides a simple, robust, and broadly applicable description of nonideal behavior in concentrated solutions of bovine serum albumin and other nonassociating proteins. J Pharm Sci. (2007) 96:3466-9. doi: 10.1002/jps.20964

19. Zhou HX, Rivas G, Minton AP. Macromolecular crowding and confinement: biochemical, biophysical, and potential physiological consequences. Ann Rev Biophys. (2008) 37:375-97. doi: 10.1146/annurev.biophys.37.032807. 125817

20. Marenduzzo D, Finan K, Cook PR. The depletion attraction: an underappreciated force driving cellular organization. J Cell Biol. (2006) 175:681-6. doi: 10.1083 /jcb. 200609066

21. Minton AP. The effect of volume occupancy upon the thermodynamic activity of proteins: some biochemical consequences. Mol Cell Biochem. (1983) 55:119-40. doi: 10.1007/BF00673707

22. Lebowitz JL, Helfand E, Praestgaard E. Scaled particle theory of fluid mixtures. J Chem Phys. (1965) 43:774-9. doi: 10.1063/1.1696842

23. Cotter MA. Hard spherocylinders in an anisotropic mean field: a simple model for a nematic liquid crystal. J Chem Phys. (1977) 66:1098-106. doi: $10.1063 / 1.434044$

24. Hall D, Mnton AP. Effects of inert volume-excluding macromolecules on protein fiber formation. II. Kinetic models for nucleated fiber growth. Biophys Chem. (2004) 107:299-316. doi: 10.1016/j.bpc.2003.09.016

25. Hall D, Edskes H. A model of amyloid's role in disease based upon fibril fracture. Biophys Chem. (2009) 145:17-28. doi: 10.1016/j.bpc.2009.08.004

26. Knowles TPJ, Waudby CA, Devlin GL, Cohen IA, Aguzzi A, Vendruscolo M, et al. An analytical solution to the kinetics of breakable filament assembly. Science (2009) 326:1533-7. doi: 10.1126/science. 1178250

27. Glabe CG. Common mechanisms of amyloid oligomer pathogenesis in degenerative disease. Neurobiol Aging (2006) 27:570-5. doi: 10.1016/j.neurobiolaging.2005.04.017

28. Marshall KE, Marchante R, Xue WF, Serpell LC. The relationship between amyloid structure and toxicity. Prion (2014) 8:1-5.

Conflict of Interest Statement: The author declares that the research was conducted in the absence of any commercial or financial relationships that could be construed as a potential conflict of interest.

Received: 09 June 2014; accepted: 24 July 2014; published online: 12 August 2014. Citation: Minton AP (2014) The effect of time-dependent macromolecular crowding on the kinetics of protein aggregation: a simple model for the onset of age-related neurodegenerative disease. Front. Phys. 2:48. doi: 10.3389/fphy.2014.00048

This article was submitted to Biophysics, a section of the journal Frontiers in Physics. Copyright (C) 2014 Minton. This is an open-access article distributed under the terms of the Creative Commons Attribution License (CC BY). The use, distribution or reproduction in other forums is permitted, provided the original author(s) or licensor are credited and that the original publication in this journal is cited, in accordance with accepted academic practice. No use, distribution or reproduction is permitted which does not comply with these terms. 